\title{
Uso de Antibióticos en Infección de Vías Urinarias en una Unidad de Primer Nivel de Atención en Salud, Bogotá, Colombia
}

\author{
Olga A. Murillo-Rojas ${ }^{1}$, Aura L. Leal-Castro ${ }^{2}$ y Javier H. Eslava-Schmalbach ${ }^{3}$ \\ ${ }^{1}$ Médica. M. Sc. Infecciones y Salud en el Trópico. Unidad de Servicios de Salud de la Universidad \\ Nacional de Colombia-UNISALUD. E-mail: oamurillor@unal.edu.co \\ 2 Médico. M. Sc. Departamento de Microbiología, Facultad de Medicina, Universidad Nacional de \\ Colombia. E-mail: allealc@unal.edu.co \\ ${ }^{3}$ Médico. M. Sc. Ph. D (candidato) Facultad de Medicina, Universidad Nacional de Colombia. E-mail: \\ jheslavas@unal.edu.co
}

Recibido 31 Octubre 2005/Enviado para Modificación 10 Abril 2006/Aceptado 28 Mayo 2006

\section{RESUMEN}

Objetivos Describir el uso de antibióticos en infección de vías urinarias, en una unidad de primer nivel de atención en salud.

Métodos Estudio observacional, descriptivo, de una cohorte histórica, basada en registros, de usuarios consultantes en la Unidad de Servicios de Salud-UNISALUD, de la Universidad Nacional de Colombia, sede Bogotá, con diagnóstico de infección de vías urinarias, entre julio de 2002 y junio de 2003.

Resultados La incidencia de infección de vías urinarias fue de 6,3\%, afectando especialmente a las mujeres con $84,4 \%$. Los signos y síntomas son los típicamente encontrados en esta entidad: $58 \%$ de los casos corresponde a infecciones bajas, $36 \%$ a inespecíficas, $4 \%$ a infecciones durante el embarazo y $2 \%$ a infecciones altas. Los uropatógenos aislados fueron: $E$. coli $(88,9 \%)$, Proteus spp. (5,1\%), Klebsiella spp. (3,7\%), Enterobacter spp. (1\%), Citrobacter spp. (1\%) y Staphylococcus saprophyticus $(0,3 \%)$. Hubo una gran variabilidad en la prescripción de esquemas antibióticos. Los de mayor consumo, en dosis diaria definida, fueron: Nitrofurantoína, Trimetoprim-sulfa y Fluoroquinolonas, frente a los cuales, los uropatógenos aislados presentaron perfiles de susceptibilidad bajos. La proporción de tratamientos empíricos con respecto a los confirmados fue de 4:1. El $98 \%$ de los casos correspondió a pacientes con un único evento, o con una o dos recurrencias.

Discusión La infección de vías urinarias presentó un comportamiento acorde al perfil epidemiológico de esta entidad patológica en la población general. La baja susceptibilidad de los microorganismos a los antibióticos 
prescritos, podría reflejar un uso inadecuado, a nivel de la comunidad institucional, induciendo presión selectiva sobre la resistencia bacteriana.

Palabras Clave: Atención ambulatoria, antibióticos, agentes antibacterianos, infección, urinaria, resistencia bacteriana a drogas (fuente: DeCS, BIREME).

\section{ABSTRACT \\ Using antibiotics in urinary tract infection in a first level of attention health care unit in Bogotá, Colombia}

Objectives Describing using antibiotics for urinary tract infection in a first level of attention health care unit.

Methods This was an observational and descriptive study of a cohort of register-based patients attending the Universidad Nacional de Colombia's Health Service Unit (UNISALUD) diagnosed as suffering urinary tract infection between July 2002 and June 2003.

Results $6,3 \%$ urinary tract infection incidence was found, especially affecting women $(84,4 \%)$. The signs and symptoms were the same as those usually found in this disease. 58\% corresponded to lower tract infection, $36 \%$ to non-specific infection, $4 \%$ to infection during pregnancy and $2 \%$ to upper tract infection. The following uropathogens were isolated: E. coli $(88.9 \%)$, Proteus spp. (5,1\%), Klebsiella spp. (3,7\%), Enterobacter spp. (1\%), Citrobacter spp. (1\%) and Staphylococcus saprophyticus $(0,3 \%)$. There was great variability in the antibiotic schemes being prescribed. Nitrofurantoin, Trimethoprim-sulfamethoxizole and Fluoroquinolones were the most prescribed, per defined daily dose; these antibiotics presented low susceptibility for the uropathogens so isolated. There was a 4:1 ratio of empirical treatment respecting confirmed ones. $98 \%$ of the cases corresponded to patients suffering from a single event or having just 1 to 2 recurrences.

Discussion The urinary tract infection studied here presented a similar epidemiological profile to that usually found in the general population. The isolated microorganism's low susceptibility to the prescribed antibiotics could reflect inappropriate use of antibiotics by the health service, inducing selective pressure on bacterial resistance.

Key Words: Out-patient care, antibiotic, anti-bacterial agent, urinary tract infection, drug resistance, bacterial (source: MeSH, NLM).

4 l uso racional de los antibióticos es una necesidad sentida en el país y el mundo entero. La Organización Mundial de la Salud OMS considera que: "El uso abusivo de los antibióticos es una de las principales causas del incremento de la resistencia bacteriana, uno de los mayores problemas de salud pública” (1). La prescripción no adecuada y abusiva de los antibióticos, la prolongación de los planes más allá de lo necesario, la aplicación de dosis 
no óptimas, la irregularidad en la toma de las drogas, son los principales factores que han llevado a que hoy la tasa de resistencia antimicrobiana sea tan elevada (2).

La infección de vías urinarias (IVU), es la infección bacteriana más común adquirida en comunidad, que afecta principalmente a la población femenina. Representa un significativo problema de salud debido a su incidencia y morbilidad, con riesgos potenciales a largo plazo en la sobrevida y calidad de vida de aquellos afectados, lo que amerita intensificar los esfuerzos en la detección temprana, el tratamiento oportuno y eficaz, y el estudio y seguimiento posteriores, especialmente en momentos y en circunstancias de alta susceptibilidad $(3,4)$.

Según el informe técnico de la división de enfermedades bacterianas y micóticas del Centro para el Control y la Prevención de Enfermedades de los Estados Unidos CDC, de octubre de 2005, alrededor de 4 millones de consultas médicas ambulatorias son por IVU, lo que representa $1 \%$ del total de consultas ambulatorias en los Estados Unidos (5). Financieramente, el costo anual estimado para la IVU, adquirida en la comunidad, en los Estados Unidos es aproximadamente U\$1,6 mil millones (6). En nuestro medio es difícil determinar exactamente la incidencia de la IVU, pues no es una entidad de reporte obligatorio. En la Unidad de Servicios de Salud-UNISALUD de la Universidad Nacional de Colombia, sede Bogotá, esta entidad patológica constituye el segundo motivo de consulta más frecuente, después de las infecciones respiratorias.

Este estudio describe el uso de antibióticos en la infección de vías urinarias, en la consulta intramural de UNISALUD.

\section{MÉTODOS}

Se realizó un estudio observacional, descriptivo, basado en registros correspondientes a una cohorte histórica de pacientes consultantes de UNISALUD, de la Universidad Nacional de Colombia, sede Bogotá, entre el 1 de julio de 2002 al 30 de junio de 2003.

Para el 2002/2003 UNISALUD, sede Bogotá, contaba con 13313 usuarios potenciales (inscritos), entre afiliados y beneficiarios, de los cuales 6 919 fueron mujeres (52 \%) y 6394 hombres (48\%).

Se tuvieron en cuenta los usuarios que acudieron a consulta médica intramural y se les diagnosticó infección de vías urinarias, durante el tiempo 
de estudio. Se consideraron los diagnósticos de cualquier tipo de infección de vías urinarias, registradas bajo el Código Internacional de Enfermedades (CIE10). Del sistema integrado de información de la Institución, el cual contiene la información sistematizada de los pacientes desde el año 2000, se seleccionaron todos los casos que cumplían con los criterios de inclusión y se extrajo la información general de los pacientes (nombre, edad, género, tipo de afiliación, peso, datos clínicos, comorbilidad y tratamiento), así como también los datos de laboratorio (parcial de orina, urocultivo y antibiograma).

Para la recolección de la información se diseñó un formato, en el que se consignaron todos los datos necesarios para el estudio. Toda la información de los formatos se digitó en una base de datos, diseñada en Excel ${ }^{\circledR}$, para realizar el proceso de análisis.

Las variables tenidas en cuenta fueron: datos generales del paciente, tipos de infección de vías urinarias, signos y síntomas, comorbilidad, microorganismos aislados, antibiogramas, antibióticos prescritos, tiempo de tratamiento, tipo de diagnóstico y recurrencias.

Se operacionalizaron los datos por medio de análisis univariado descriptivo, presentados a través de tablas y gráficas de distribución de frecuencias para cada una de las variables.

Se estimo la incidencia de la entidad y la distribución de frecuencias de: los tipos de IVU, signos y síntomas, espectro etiológico teniendo en cuenta únicamente los urocultivos positivos, perfil de susceptibilidad, antibióticos prescritos, dosificación y consumo de antibióticos en Dosis Diaria Definida/1.000 usuarios-día (DDD, según la Anatomical Therapeutic Chemical Classification/Organización Mundial de la Salud) (7), tiempos de tratamiento y recurrencias.

Para la interpretación de los antibiogramas se siguieron las normas de la NCCLS (8). Reconociendo que el reporte del perfil de susceptibilidad solo contemplaba las categorías sensible y resistente, la susceptibilidad disminuida correspondiente a la categoría intermedia, se incluyo dentro de la categoría resistente.

\section{RESULTADOS}

De los 13313 usuarios potenciales, 10996 acudieron a consulta médica durante el tiempo de estudio. De estos usuarios consultantes, 694 tuvieron 
diagnóstico de infección de vías urinarias, los cuales corresponden a la muestra del estudio. De los usuarios diagnosticados con IVU, la mayoría correspondieron a mujeres (84,4 \%), con predominancia en el grupo de edad entre 15 y 44 años (Tabla 1).

Tabla 1. Distribución porcentual población con diagnóstico de IVU por grupos de edad y género UNISALUD Bogotá, julio 2002 a junio 2003

\begin{tabular}{ccccccc}
\hline \multirow{2}{*}{$\begin{array}{c}\text { Grupos de } \\
\text { edad. Años }\end{array}$} & \multicolumn{2}{c}{ Mujeres } & \multicolumn{2}{c}{ Hombres } & \multicolumn{3}{c}{ Total } \\
\cline { 2 - 7 } & No. & $\%$ & No. & $\%$ & No. & $\%$ \\
\hline $0-5$ & 12 & 1,8 & 2 & 0,2 & 14 & 2 \\
$6-14$ & 24 & 3,4 & 4 & 0,8 & 28 & 4,2 \\
$15-44$ & 220 & 31,8 & 27 & 3,9 & 247 & 35,7 \\
$45-59$ & 153 & 22 & 31 & 4,4 & 184 & 26,4 \\
60 o más & 177 & 25,4 & 44 & 6,3 & 221 & 31,7 \\
\hline Total & 586 & 84,4 & 108 & 15,6 & 694 & 100 \\
\hline
\end{tabular}

Fuente: Base de datos SAMI UNISALUD Bogotá 2002-2003

De los usuarios diagnosticados con IVU, 349 (50,3 \%) correspondieron a afiliados cotizantes, de los cuales 199 (57 \%) eran laboralmente activos y 165 (83 \%) de los laboralmente activos fueron mujeres.

La incidencia acumulada (IA) o riesgo de infección de vías urinarias, en UNISALUD sede Bogotá, entre julio de 2002 a junio de 2003, fue de 6,3 \%. En mujeres la incidencia acumulada de IVU fue de 9,8 \% y en hombres de 2,2 \% (Tabla 2).

Tabla 2. Incidencia acumulada de IVU por grupos de edad y género

\begin{tabular}{|c|c|c|}
\hline \multirow{2}{*}{ Grupos de edad (años) } & \multicolumn{2}{|c|}{ Incidencia acumulada \% } \\
\hline & Mujeres $(n=586)$ & Hombres $(n=108)$ \\
\hline $0-5$ & 5,3 & 0,8 \\
\hline $6-14$ & 2,9 & 0,5 \\
\hline $15-44$ & 12 & 2,2 \\
\hline $45-59$ & 8,6 & 2,2 \\
\hline 60 o más & 13,5 & 3,4 \\
\hline
\end{tabular}

Fuente: Base de datos SAMI UNISALUD Bogotá 2002-2003 
Los signos y síntomas que conformaron el cuadro clínico de la IVU fueron: disuria $45 \%$ de los casos; polaquiuria $34 \%$; dolor abdominal $29 \%$; lumbalgia $19 \%$; fiebre $11 \%$; escalofrío $10 \%$; tenesmo vesical $7 \%$; urgencia urinaria $7 \%$; cefalea $6 \%$; hematuria $5 \%$; náuseas $4 \%$; orina olorosa 3 $\%$; nicturia $3 \%$; vómito $3 \%$ y otros (sonda vesical, incontinencia urinaria, molestia en genitales externos masculinos y edemas) $4 \%$.

Las entidades patológicas más frecuentemente asociadas a la IVU fueron: sobrepeso 26 \% de los casos; hipertensión arterial 25 \%; hipotiroidismo 17 $\%$; alteraciones funcionales de las vías urinarias $6 \%$; prostatismo $5 \%$; alteraciones anatómicas de las vías urinarias $4 \%$; diabetes mellitus tipo II $4 \%$; cáncer $2 \%$; secuelas de enfermedad cerebro vascular $2 \%$ y la insuficiencia renal crónica $1 \%$.

El diagnóstico de IVU baja se hizo en 58 \% de los casos, 36 \% a IVU inespecíficas, $4 \%$ a IVU durante el embarazo y $2 \%$ a IVU altas. El diagnóstico de IVU bajas incluyeron básicamente las cistitis con $95 \%$, el $5 \%$ restante correspondieron a uretritis. 87,7 \% de las cistitis fueron mujeres y 85,7 $\%$ hombres. De las IVU inespecíficas, 83 \% fueron mujeres y $17 \%$ hombres.

De los 694 pacientes diagnosticados con IVU a 304 (44 \%) se les realizaron urocultivos. El total de urocultivos realizados fue de 470 , de los cuales fueron positivos 296 (63\%) y negativos 174 (37\%). El número de urocultivos por paciente osciló entre 1 y 6; con 1 urocultivo 65,8 \%; con 2 urocultivos 23,3\%, con 3 urocultivos 4,6 \%; con 4 urocultivos 3,6 \%, con 5 urocultivos $1,9 \%$ y con 6 urocultivos $0,65 \%$ de los casos.

En los urocultivos positivos se aislaron los siguientes microorganismos: Escherichia coli (88,9 \%), Proteus spp. (5,1\%), Klebsiella spp. (3,7\%), Enterobacter spp. (1\%), Citrobacter spp. (1\%), y Staphylococcus saprophyticus $(0,3 \%)$.

El perfil de susceptibilidad encontrado para Escherichia coli respecto a los agentes utilizados en el tratamiento de la IVU en la población de estudio, mostró las tasas de resistencia más elevadas para el grupo de agentes tipo Beta-lactámicos, especialmente para Ampicilina y Amoxicilina, seguidos por Trimetoprim-sulfa y Fluoroquinolonas. Las tasas de resistencia más bajas se observaron para agentes como la Nitrofurantoína y Aminoglucósidos (Tabla 3).

Aunque los otros uropatógenos aislados (Proteus spp., Klebsiella spp., Enterobacter spp. y Citrobacter spp.), no tuvieron una participación signifi- 
cativa en este estudio, vale la pena resaltar las altas tasas de resistencia que presentaron al Trimetoprim-sulfa, las cuales oscilaron entre 42,9 \% y 66,7 \% y a la Amoxicilina con tasas de resistencia entre 50 \% y $100 \%$; para la Cefalotina se observaron rangos muy amplios de susceptibilidad; para la Nitrofurantoína se encontraron resistencias entre $0 \%$ y $25 \%$ y para la Gentamicina tasa de resistencia de $0 \%$. El único caso de Staphylococcus saprophyticus mostró tasas de resistencia extrapoladas, por ejemplo de $0 \%$ a Penicilinas más inhibidores de Betalactamasas y Cefalosporinas de primera generación, y de 100 \% de resistencia a Ciprofloxacina, Gentamicina y Trimetoprim-sulfa. El total de tratamientos con antibiótico para IVU, fue de 704.

Tabla 3. Perfil de resistencia para Escherichia coli UNISALUD Bogotá, julio 2002 a junio 2003

\begin{tabular}{cccc} 
& $(\mathrm{n}=263)$ & & \\
\hline Antibiótico* & Aislamientos probados & Resistentes & $\%$ \\
\hline Ampicilina & 37 & 23 & 62,2 \\
\hline Ampicilina-Sulbactam & 88 & 11 & 12,5 \\
\hline Amoxicilina & 36 & 22 & 61,1 \\
\hline Amoxicilina-Clavulánico & 103 & 9 & 8,7 \\
\hline Cefalotina & 196 & 48 & 24,5 \\
\hline Ciprofloxacina & 216 & 37 & 17,1 \\
\hline Norfloxacina & 178 & 39 & 21,9 \\
Amikacina & 12 & 0 & 0,0 \\
\hline Gentamicina & 235 & 18 & 7,7 \\
\hline Nitrofurantoína & 233 & 10 & 4,3 \\
\hline Trimetoprim-Sulfa & 229 & 94 & 41,0 \\
\hline Fuente: base de datos WINSISLAB UNISALUD Bogotá 2002 - 2003 \\
*Antibióticos utilizados en los antibiogramas cuando se aisló E. coli &
\end{tabular}

Se observó una gran variabilidad en las dosis prescritas por antibiótico, tanto para adultos como para menores de 13 años. El tiempo de prescripción, en casos agudos, osciló entre 1 y 20 días; para los tratamientos con fines profilácticos de 30 o más días.

Los antibióticos de mayor consumo en DDD fueron Trimetoprim-sulfa y Nitrofurantoína, los cuales fueron prescritos para fines profilácticos, seguidos por las Fluoroquinolonas. 
Se prescribieron 704 tratamientos, 553 (79 \%) por sospecha de IVU y 151 (21 \%) por IVU confirmadas. De los pacientes que recibieron tratamientos por sospecha de IVU, 119 (20\%) tuvieron urocultivos y se les prescribió el antibiótico al momento del diagnóstico; 71 de estos urocultivos fueron positivos y 48 fueron negativos, por lo tanto, $60 \%$ confirmaron la IVU y $40 \%$ la descartaron. En 63 de los casos con urocultivos positivos (89\%), se acertó prescribir el antibiótico según los antibiogramas. A los 434 casos restantes por sospecha de IVU (78 \%), no se les realizaron urocultivos al momento del diagnóstico.

En la revisión de las historias clínicas de los casos por sospecha de IVU tratados con antibiótico se encontró: 4 casos con respuesta clínica y confirmación de la erradicación del microorganismo In Vitro; 12 casos sin respuesta clínica (estos 12 casos tuvieron el siguiente comportamiento: 4 sin erradicación del microorganismo y 2 casos, en un mismo paciente, con erradicación a pesar de continuar sintomático, los 6 casos restantes no tuvieron comprobación In Vitro). De los 553 casos restantes de sospecha de IVU, 537 (97\%) no tuvieron evidencia clara de respuesta al tratamiento, pues no se confirmó ni clínica ni bacteriológicamente en el historial clínico.

De los 151 casos con tratamiento antibiótico en IVU confirmadas, 145 (96 \%) no contaron con una confirmación clara de la respuesta al tratamiento, ni clínica ni bacteriológica. De los 6 casos restantes, 4 no tuvieron respuesta clínica ni erradicación del microorganismo a pesar de que el antibiótico utilizado se ajustara al reporte de susceptibilidad y 2 casos, a pesar de obtenerse respuesta clínica, no se obtuvo erradicación del microorganismo In Vitro pos tratamiento.

De los 694 pacientes con diagnóstico de IVU, 138 (20 \%) presentaron recurrencias. El número de recurrencias por paciente osciló entre 1 y 5; 109 pacientes presentaron 1 (79 \%), 19 presentaron 2 (13,8 \%), 5 presentaron 3 (3,6 \%), 2 presentaron $4(1,4 \%)$ y 3 presentaron 5 (2,1\%), para un total de 185 recurrencias. De lo anterior se deduce que durante el tiempo de estudio, 556 (80 \%) pacientes fueron diagnosticados una sola vez con IVU y 128 con 1 a 2 recurrencias.

\section{DISCUSIÓN}

La infección de vías urinarias, en una unidad de primer nivel de atención en salud, en Bogotá, Colombia, presenta un comportamiento acorde al perfil epidemiológico de esta entidad patológica descrito en la población general (3-5). 
Los signos y síntomas que conforman el cuadro clínico de la IVU, caracterizan una gran variabilidad clínica e inespecífica de esta entidad patológica $(9,10)$. La comorbilidad asociada con mayor frecuencia (sobrepeso, hipertensión arterial e hipotiroidismo) no tiene una relación etiopatogénica clara con esta entidad patológica; en cambio, los diagnósticos encontrados con menor frecuencia (alteraciones funcionales y anatómicas de las vías urinarias, diabetes mellitus tipo II, cáncer, secuelas de enfermedad cerebro vascular e insuficiencia renal crónica) sí aumentan el riesgo de IVU $(5,11)$.

Los tipos de IVU más frecuentemente diagnosticados (baja e inespecífica) reflejan el nivel de complejidad clínica de la entidad que se presenta y se maneja en UNISALUD sede Bogotá; además, el porcentaje de IVU inespecíficas, puede estar reflejando la proporción de casos con clínica y etiología no muy bien definidas, los cuales requieren soportes paraclínicos complementarios y muy seguramente valoración especializada $(3,9,12)$.

Con relación a la susceptibilidad disminuida, correspondiente a la categoría intermedia, la cual no se reporta en los antibiogramas de los urocultivos analizados, tiene implicaciones clínicas en la aplicabilidad de las concentraciones fisiológicas que se pueden obtener del agente antimicrobiano en las diferentes partes del cuerpo (8).

Al correlacionar el perfil de susceptibilidad de la Escherichia coli encontrado en este estudio con estudios de resistencia antimicrobiana en uropatógenos, frente a agentes como: Beta-lactámicos, Trimetoprim-sulfa, Fluoroquinolonas y Nitrofurantoína, podemos decir que en este orden, las tasas de resistencia es el mismo; sin embargo, las tasas de resistencia encontradas en la literatura foránea no son tan altas en comparación con las encontradas en este estudio $(13,14,15)$. A nivel local, desafortunadamente no se cuenta con estudios sobre susceptibilidad en uropatógenos, en la comunidad. De cualquier manera, este estudio muestra una disminución de la susceptibilidad de la Escherichia coli a los antibióticos más utilizados en el tratamiento de la IVU, lo que puede estar reflejando un uso inadecuado de estos agentes a nivel de comunidad, que estén provocando una presión selectiva sobre el comportamiento de la resistencia bacteriana. Es de destacar que al momento de la realización de este estudio, en Colombia no había una norma que restringiera el uso y consumo de antibióticos en la comunidad, por lo que eran de venta libre.

Al revisar la literatura correspondiente, los antibióticos más formulados para el tratamiento de la IVU, en este estudio, corresponden a los recomendados, bien sea como primeras o segundas opciones, aunque vale la pena te- 
ner en cuenta que las Fluoroquinolonas son agentes de segunda opción $(9,16,17)$.

El cálculo del consumo de medicamentos en DDD, unidad de medida internacionalmente reconocida (7), además de reflejar el impacto económico que pueda tener el uso de antibióticos en el tratamiento de la IVU en la institución de referencia, también puede ser útil para valorar la presión selectiva del consumo de antibióticos con la resistencia bacteriana.

$\mathrm{Al}$ analizar la antibioticoterapia podemos evidenciar una gran variabilidad de esquemas, observada tanto en la elección del tipo de antibiótico, como en las dosificaciones y tiempos de tratamiento, lo que no va de acuerdo con las recomendaciones de la Sociedad Americana de Enfermedades Infecciosas $(3,9,10,16,17)$.

La proporción de tratamientos empíricos prescritos para IVU, con respecto a los confirmados, es de 4 a 1 , lo que puede estar sugiriendo gastos injustificados en antibióticos, y además estar contribuyendo en la aparición de resistencia bacteriana. En consecuencia con lo anterior, se puede recurrir a pruebas rápidas de diagnóstico, como por ejemplo: sedimento urinario, tinción Gram, tiras reactivas, que orienten el tratamiento, mientras se tiene el reporte del urocultivo (18).

Con respecto a los tratamientos con antibiótico prescritos en IVU confirmadas, en 96 \% de estos casos no hay una confirmación clínica ni bacteriológica al tratamiento, lo que no nos permite hacer un seguimiento más juicioso de la respuesta a los respectivos tratamientos.

Al considerar que la población con un único evento de IVU y la población con 1 a 2 recurrencias corresponde al $98 \%$, sugiere que el protocolo de manejo de pacientes con IVU en UNISALUD sede Bogotá, debe dirigirse de manera especial a IVU aisladas (primo infección o aislada de previas infecciones por lo menos 6 meses) (3).

Los resultados que se obtuvieron con la realización de este estudio, además de ofrecer el perfil epidemiológico de la infección de vías urinarias (IVU), dan cuenta de un patrón general del uso de antibióticos, en una entidad infecciosa, describiendo todo el proceso que de éste se genera, desde el momento del diagnóstico, tanto clínico como bacteriológico, hasta la instauración y seguimiento del tratamiento. En dichos resultados se observan tanto debilidades como fortalezas para, desde allí, plantear lineamientos claros sobre las conductas que se deben seguir en el manejo integral de esta entidad 
patológica desde programas de promoción y prevención, así como también protocolos para la realización de pruebas de laboratorio, protocolos de manejo clínico y sistema de vigilancia y control de uso de antibióticos y resistencia bacteriana.

\section{REFERENCIAS}

1. Avorn JL, Barret JF, Davey PG, McEwen SA, O’Brian FF, Levy SB. Antibiotic resistance synthesis of recommendations by expert policy grups. World Health Organization. Boston; 2001.

2. Centers for Desease Control and Prevention, Food and Drug Administration and the National Institutes of Health. A Public Health Action Plan to Combat Antimicrobial Resistance. Interagency Task Force. Co-chairs; 1999.

3. Safir MH, Schaeffer AJ. Urinary tract infection: simple and complicated. AUA Update series 1997; 16(10).

4. Malo Rodríguez G, Echeverry Raad J, Iragorri S, Gastelbondo R. Infección urinaria en niños menores de dos años. Guía de práctica clínica. Sociedad Colombiana de Urología, 1999.

5. Centers for Disease Control and Prevention. Urinary tract infections. Technical information. Division of bacterial and mycotic diseases. October 25, 2005. [Internet]. Disponible en: http://www.cdc.gov/ncidod/dbmd/diseaseinfo/ urinarytractinfections_t.htm. Consultado Junio de 2006.

6. Foxman B. Epidemiology of urinary tract infections: incidence, morbidity, and economic costs. Am J Med 2002; 113(1): 5-13.

7. WHO. Collaborating Centre for Drug Statistics Methodology. ATC/DDD applications 2003. [Internet]. Disponible en: http://www.whocc.no/atcddd/. Consultado Noviembre de 2003.

8. Ferraro MJ, Craig WA, Dudley MN, Eliopoulos G, Hecht DW, Hindler JF, et al. Performance Standards for Antimicrobial Susceptibility Testing; Twelfth informational supplement, 2002.

9. Fihn SD. Acute uncomplicated urinary tract infection in women. New England Journal of Medicine 2003; 349(3): 259-266.

10. Pigrau C, Horcajada JC, Cartón JA, Pujol M. Protocolos Clínicos. S.E.I.M.C. IV. Infección Urinaria. [Internet]. Disponible en: http://www.seimc.org/ protocolos/clinicos/proto4.htm. Consultado Noviembre de 2003.

11. Karlowsky JA, Kelly LJ, Thornsberry C, Jones ME, Sahml DF. Trends in Antimicrobial Resistance among Urinary Tract Infection Isolates of Escherichia coli from Femal Outpatients in the United States. Antimicrobial Agents and Chemotherapy. 2002; 46(8): 2540-2545.

12. Ronald A. The etiology of urinary tract infection: traditional and emerging pathogens. American Journal of Medicine 2002; 113(1): 14-19.

13. Gupta K, Scholes D, Stamm WE. Increasing prevalence of antimicrobial resistance among uropathogens causing acute uncomplicated cystitis in women. JAMA 1999; 281(8): 736-738.

14. Gupta K. Addressing antibiotic resistance. Am J Med 2002; 113(1): 29-34. 
15. Gupta K, Hooton TM, Stamm WE. Increasing antimicrobial resistance and the management of uncomplicated community-Acquired urinary tract infections. Ann Intern Med 2001; 135: 41-50.

16. Warren JW, Abrutyn E, Hebel JR, Johnson JR, Schaeffer AJ, Stamm WE. Guidelines for antimicrobial treatment of uncomplicated acute bacterial cystitis and acute pyelonephritis in women. Guidelines from the infectious diseases society of America. Clinical Infectious Diseases 1999; 29: 745758.

17. Nicolle LE. Urinary tract infection: traditional pharmacologic therapies. Am J Med 2002; 113(1): 35-44.

18. Bent S, Saint S. The optimal use of diagnostic testing in women with acute uncomplicated cystitis. Am J Med 2002; 113(1): 20-28. 